\title{
A Topology Control Algorithm Based on Pass Loss for Wireless Sensor Network
}

\author{
Nanxi Luo \\ Department of Communication College \\ Chongqing university of Posts and Telecommunications \\ Chongqing, China \\ E-mail: luonanxii@163.com
}

\author{
Jie Bao \\ Department of Communication College \\ Chongqing university of Posts and Telecommunications \\ Chongqing, China \\ e-mail: baoj@cqupt.edu.cn
}

\begin{abstract}
We present PLTC,a distributed topology control algorithm that based on pass-loss for wireless sensor network .In contrast to the algorithm based on RSSI,it can be applied on a heterogeneous network and nodes with different transmitting power.In this paper,we discuss the shortcomings of other parameters used to determine the link quality and present PLTC.We evaluate PLTC by analytic proofs and simulation results. The result shows it has good performance on bounded degree,connectivity and planarity.
\end{abstract}

Keywords-WSN; topology control;Path loss; link quality

\section{INTRODUCTION}

The wireless sensor network consists of spatially distributed autonomous sensors to monitor physical or environmental conditions, such as temperature, sound, pressure, etc. Each node in WSN has typically several parts: a radio transceiver, a microcontroller, an electronic circuit for interfacing with the sensors and an energy source, usually a battery.WSN is energy constrained and resource constrained,demanding that the energy and lifetime of the note is the key factor when design any mechanisms and algorithms.

The topology control for WSN can reduce the redundancy connection, thus reduce the energy cost.A lot of algorithms feature a sequence of properties, but these algorithms also need some information like location and distance which required GPS or other location tracking techniques. In WSN,the topology control algorithm must satisfies four requirements: first of all,the algorithms should run in

distributed manner, so they can be implemented in large networks. Second, topology construction algorithms must have a low computational and message complexity, so they can be efficiently run in computationally weak devices.Third,the algorithms are able to run without the help of additional hardware like GPS devices or other localization mechanisms. Finally, the topology control algorithm must produce a connected network that will cover the area with a minimum number of nodes and keep the network active the longest possible time.

Early algorithm with best practicability for WSN is $\mathrm{XTC}^{[1]}$.XTC uses the received signal strength indicator(RSSI)of the radio on the nodes as a linkmetic, which can be easily obtained in a sensor nodes.

XTC is practical,but still it has some limits and unrealistic assumptions.First,the RSSI model can not be applied on heterogeneous network,cause different type of can not reflect the real distance and link quality between nodes.Second,

In response to the problems above, we proposed WSN topology control algorithm based on path loss.Path loss can make accurate estimate on link quality.It has some advantages over RSSI.First, if nodes are stationary or slowly moved,the path loss can be defined within a range.Second,path loss only depend on the environment, and can be applied on a heterogeneous network and nodes with different transmitting power.

\section{THE PROPOSED NETWORK SELECTING}

\section{ALGORITHM}

\section{A. Definition of link quality}

Generally speaking,there are three parameter to reflect the link quality,packet reception rate(PRR),link quality indictor(LQI) and received signal strength indictor(RSSI).

PRR is defined as the proportion of the average number of correct received packets to send in a period time.PRR is the most accurate parameter of link quality, but it is difficult to measure for most of the WSN mode. The RSSI value is the result of averaging the received power over eight symbol periods(128ms)as specified by IEEE 802.15.4[2].It is easy to measure in most WSN nodes.Take CC2530 system-on-chip node for instance,The CC2530 radio has a built-in received signal-strength indication (RSSI), which calculates an 8-bit signed digital value that can be read from a register or automatically appended to received frames. The link quality indication (LQI) is a measurement of the strength and/or quality of the received frame as defined by the IEEE 802.15.4 standard. For CC2530,the radio does not provide an LQI Value directly, in fact the RSSI value can be used by the MAC software to calculate the LQI value or a combination of RSSI and correlation values may also be used to generate the LQI value.

Hao Xiao-chen and his group have analysed the relationship between PRR and RSSI in[3], and draw the conclusion that the PRR increases exponentially with the increase of RSSI.In most cases,LQI is calculated based on RSSI.

Based on the analysis above,we can see that the RSSI is the most practical and easy way to determine the link quality.But still RSSI has some limits when used in the 
algorithm.First,the RSSI fluctuation results that the RSSI values are not a monotonously decreasing function of the distance.Second, in a real scenario as time goes on,nodes may differ in transmission power, and in a heterogeneous network, different nodes have different transmission power,in those situation, use RSSI to determine the distance may cause some mistakes.According to [3],RSSI is a monotonously increasing function of the transmission power, that means, RSSI is not only related to the link quality but also states of nodes.

Pass loss which is only related to the distance and environment between nodes, is more suitable to determine the link quality, and more suitable for topology control of heterogeneous network with different transmission power.First,pass loss don't require all the nodes have the same transmission power, it has broader applicability than RSSI.Second, if nodes are stationary or slowly moved, the path loss does not fluctuate as much as RSSI.Finally, as the environment remains unchanged,bi-directional link quality is symmetrical.

\section{B. Algorithm description}

In this section,we describe our pass-loss based topology control algorithm .The algorithm consists of four main steps:

I. Neighbor discovering

II. Neighbor ID exchange and ordering

III. Neighbor order exchange

IV. link selection

The algorithm is executed at all nodes of the network, the description shown next is from the perspective of node $u$. The pseudocode is showing in the next box.

In the first step each network node $\mathrm{u}$ broadcast a Hello message include ID number and value of the $\max$ transmission power $p_{m}$.

In the second step, when node $u$ received the Hello message,compute the pass loss between $u$ and $v$.

$$
p_{l}=p_{m}-r s s
$$

Where rss is the RSSI measured $u$ by $v, p_{l}$ is the direct communication pass loss between $u$ and $v$.After $u$ add node $v$ to its neighbor list $\mathrm{L}$ and form a initial network graph $\mathrm{G}, \mathrm{u}$ computes a total order of pass loss $\prec_{u}$ over all its neighbors in the network graph G.The Process neighbor exchange is correspond to this step.The $\prec_{u}$ is ordered with respect to decreasing link quality. The smaller the $p_{l}$ is,the higher it ranked in the order.A neighbor $w$ ranked higher than $v$ in the order is denoted $W \prec_{u} v$.This neighbor order reflects much more of the link quality than RSSI or PRR,and it is corresponds to the order of the Euclidean distance from $u$.

In the third step,the neighbor order information is exchanged among all the neighbors.Node $u$ broadcast its order while receiving the orders computed by all of the neighbors.The Process order exchange show in the pseudocode correspond to this step.u will exchange its own order with all the neighbors formed in the second step.
In the forth step,Based on the order information,node $u$ locally select those neighbor nodes which will form its neighborhood in the resulting topology control graph.The main idea is Node $u$ traverse $\prec_{u}$ with increasing pass loss, choose the neighbor node with lower pass loss first and choose the node with higher pass loss later.The Msg_handler linkselection(node $\mathrm{u}$, list $\prec_{u}$ ) correspond to this step.

PLTC Algorithm(for node $u$ )

1: Process neighbor exchange

2: $\quad$ for (each node $u$ ) do

3: $\quad$ while (receive Hello from $v$ ) do

4: $\quad$ if $(v$ is not in the neighbor list $L)$ do

5: $\quad$ add $v$ into $L$

6: $\quad p_{l}(v)=p_{\max }(v)-\operatorname{rss}_{u}(v)$

7: $\quad$ end if

8: $\quad$ end while

9: end for

10: end process

1: process order exchange

2: $\quad$ for (each node $u$ ) do

3: if(L not empty )do

4: $v=v_{i}$

5: $\quad \operatorname{send}\left(v, \operatorname{orderlist}\left(u, \prec_{u}\right)\right)$

6: $\quad$ remove $v_{i}$ from $L$

7: $\quad$ end if

8: end for

9: end process

1: msg_handler link selection(node $u$, list $\prec_{u}$ )

2: $\quad$ remove $v$ from $L$

3: $\quad$ if $\left(\exists w: \quad w \prec_{u} v\right.$ and $\left.w \prec_{v} v\right)$ then

4: $\quad$ inform higher layer that $v$ is selected

5: $\quad$ connect $v$

6: else

7: $\quad$ inform higher layer that $v$ is removed

8: $\quad$ disconnect $v$

9: end if

10: end msg_handler

\section{ANALYSIS AND SIMULATION RESULTS}

\section{A. Algorithm Analysis}

Theorem 3.1(connectivity)If two nodes are connected in $\mathrm{G}(\mathrm{V}, \mathrm{E})$ they are connected in $\mathrm{G}_{\mathrm{PLTC}}(\mathrm{V}, \mathrm{E})$.

Proof:Assume two nodes $u, v \in \mathrm{V}$, if they are connected in $\mathrm{G}(\mathrm{V}, \mathrm{E})$, then there lies a path connect the node $u$ and $v$.According to the algorithm,since the link selection is 
selecting the link which is already exist in the previous steps.In the step I and II,the proximity graph $\mathrm{G}(\mathrm{V}, \mathrm{E})$ was formed.So in the $\mathrm{G}_{\mathrm{PLTC}}(\mathrm{V}, \mathrm{E}), u$ and $v$ is connected.

Theorem 3.2(planarity) $G_{P L T C}$ is planner, it contains no intersecting edges.

Proof:Assume in the $\mathrm{G}_{\text {PLTC }}$ edge $(u, v)$ and $(w, x)$ intersect with each other,as shown in the figure 1.In situation 1,as shown in the upper graph, the quadrangle $u w v x$ at least has one angle bigger than $\pi / 2$,take the angle $\alpha$ for instance,so edge $|w x|>|w v|$ and $|w x|>|v x|$.In situation 2,as shown in the lower graph, $\alpha=\pi / 2,|w x|>|w v|$ and $|w x|>|v x|$.when node $w$ consider the neighbor,we have $v \prec_{w} X$. So node $w$ will connect $v$ and disconnect $x$, that is contradict to the assumption that edge $|w x|$ is existed.

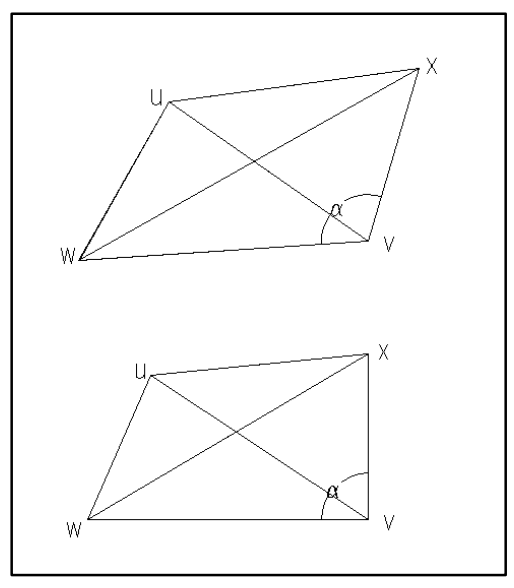

Figure 1. Proof of the theorem 3.2

\section{B. Simulation Result}

In this section we present the simulation results of the topology control graph $\mathrm{G}_{\text {PLTC. }}$ In order to model the physical wireless sensor network in our evaluation we adopt the Unit Disk Graph definition, in which an edge exists if and only if its Euclidean length is less than one unit.

We compare the topology structure before and after excise the PLTC algorithm by randomly and uniformly placing 50 sensor nodes in a $250 \mathrm{~m} \times 250 \mathrm{~m}$ square field. In order to test the properties on heterogeneous network and nodes with different transmitting power,we placed two kinds of nodes, their max transmission range are 20 meters and 30 meters. The result is shown in figure 2.The upper figure shows the topology structure without topology control.The lower figure shows the structure exciting the PLTC on each node.

The bounded degree property having been shown in figure 3.The degree of a node in a wireless sensor network is the number of connections or edges the node has to other nodes. The excessive node degrees cause propagation interference and collisions between the signals, and consume large amounts of energy. Low node degrees causes extension of transmission path and also increase the whole power consumption. The $k$-Neigh ${ }^{[4]}$ algorithm proved when nodes between $50-500,6$ is a node degree that allow at least $95 \%$ nodes connected.We implanted PLTC on 20-100 randomly deployed nodes,and counted the average node degree.The result shows in figure 3 that the node degree is slightly higher than XTC.The higher degree can provide better connectivity.
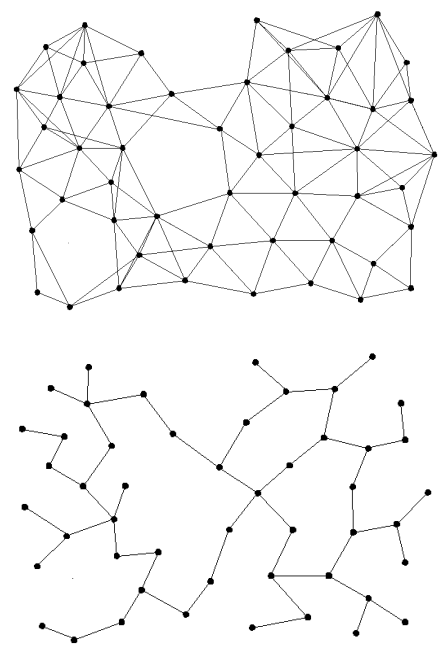

Figure 2. Comparison of topologies' structure

\section{CONCLUSION}

An distributed topology control algorithm based on the pass loss is proposed.Compared to previous algorithm has three advantages.First,it can be applied on a heterogeneous network and nodes with different transmitting power.Second, if nodes are stationary or slowly moved, the path loss can be defined within a range.Third, because the environment around two nodes are relatively unchanged, the bi-path loss have good properties in symmetry.

In this paper, we discuss the the three parameters used to determine the link quality and Euclidean distance,Based on the pass loss we present PLTC.We evaluate PLTC by analytic proofs and simulation results. The result shows it has good performance on bounded degree,connectivity and planarity.

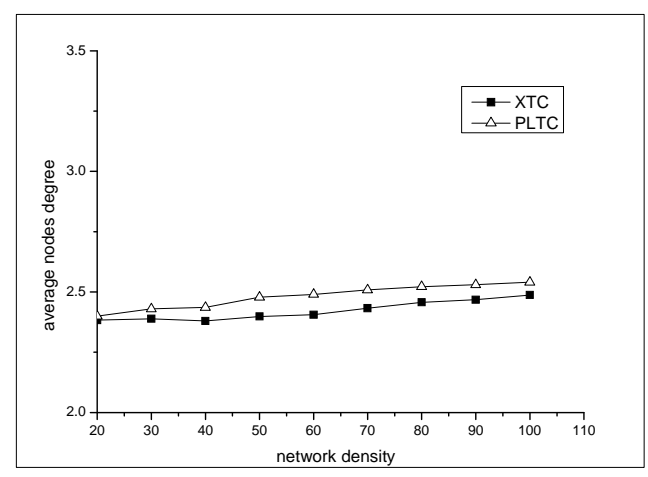

Figure 3. Average nodes' degree 


\section{REFERENCES}

[1] Roger Wattenhofer and Aaron Zollinger.XTC: A Practical Topology Control Algorithm for Ad-Hoc Networks.IPDPS'04.

[2] IEEE Std.802.15.4-2006:Wireless Medium Access Control(MAC) and Physical Layer(PHY)specifications for Low Rate Wireless Personal Area Networks(LR-WPANs).

[3] HAO Xiao-Chen, DOU Jing-Jing,LIU Bin.PathLoss Based Distributed topology Control Algorithm for Wireless Sensor Networks.Journal of Software ,Vol.20,No.12,December 2009,pp.3213-3222.

[4] Blough DM.,Leoncini M,Resta G,Santi P.The k-neighbors approach to interference bounded and symmetric topology control in ad hoc networks.IEEE Trans.on Mobile Computing,2006,5(9):1267-1282

[5] ZHANG Xue, LU Sang-Lu, CHEN Gui-Hai, CHEN Dao-Xu, XIE Li.Topology Control for Wireless Sensor Networks.Journal of Software, Vol.18, No.4, April 2007, pp.943 - 954.
[6] HUANG Man, CHENG Liang-lun.Energy-aware Topology Control Algorithm of Wireless Sensor Network Based on Path loss.Computer Engineering,March 2012,pages 92-94.

[7] LU Gang,ZHou MigTian,NIU xinzheng,SHE Kun,TANG Yong,QIN Ke.A Survey of Proximity Graph in Wireless Network.Journal of Software,Vol.19,No.4,April 2008,pp.888-911.

[8] R.Wattenhofer,L.Li, P.Bahl,Y.M.Wang.Distributed Topology Control for Power Efficient Operation in Multihop Wireless Ad Hoc Networks. In Proc.of the 20thAnnual Joint Conference of the IEEE Computer and Communications Societies (INFOCOM), 2001.

[9] Li N,Hou JC.Topology control in heterogeneous wireless networks:Problems and solutions.In:Proc.of the IEEE Conf. on Computer Communications (INFOCOM).New York:IEEE Press,2004.232-243.

[10] CC253x System-on-Chip Solution for2.4-GHz IEEE 02.15.4 and ZigBee Applications User's Guide.

[11] Olivier V;Pascal F;Maher H MPEG-2 video services over packet networks:Joint effect on encoding rate and data loss on user-oriented QoS 1998. 